\title{
Robust Vibration Control Based on Rigid-Body State Observer for Modular Joints
}

\author{
Qiang Xin ${ }^{1,2}$, , Chongchong Wang ${ }^{2}$, Chin-Yin Chen ${ }^{2, *}$, Guilin Yang ${ }^{2}$ and Long Chen ${ }^{3}(\mathbb{D}$ \\ 1 College of Materials Sciences and Opto-Electronic Technology, University of Chinese Academy of Sciences, \\ Beijing 100049, China; xinqiang@nimte.ac.cn \\ 2 Zhejiang Key Laboratory of Robotics and Intelligent Manufacturing Equipment Technology, Ningbo Institute \\ of Materials Technology and Engineering, Chinese Academy of Sciences, Ningbo 315201, China; \\ wangchongchong@nimte.ac.cn (C.W.); glyang@nimte.ac.cn (G.Y.) \\ 3 Faculty of Science and Technology, University of Macau, Macau 999078, China; longchen@um.edu.mo \\ * Correspondence: chenchinyin@nimte.ac.cn; Tel.: +86-0574-8760-2663
}

Citation: Xin, Q.; Wang, C.; Chen,

C.-Y.; Yang, G.; Chen, L. Robust

Vibration Control Based on

Rigid-Body State Observer for

Modular Joints. Machines 2021, 9, 194.

https://doi.org/10.3390/

machines 9090194

Academic Editor: Dan Zhang

Received: 30 July 2021

Accepted: 7 September 2021

Published: 10 September 2021

Publisher's Note: MDPI stays neutral with regard to jurisdictional claims in published maps and institutional affiliations.

Copyright: (c) 2021 by the authors. Licensee MDPI, Basel, Switzerland. This article is an open access article distributed under the terms and conditions of the Creative Commons Attribution (CC BY) license (https:/ / creativecommons.org/licenses/by/ $4.0 /)$.

\begin{abstract}
The vibration caused by resonance modes frequently occurs during acceleration and deceleration of the modular joint integrated with flexible harmonic drive. The conventional equivalent rigid-body velocity method with observer can suppress the residual vibration induced by resonant frequency but has poor robustness to model uncertainties and external disturbances. Moreover, it cannot eliminate the torque ripple caused by the harmonic drive during low-speed uniform motion, reducing the velocity tracking accuracy. Hence, a velocity controller with a rigid-body state observer and an adjustable damper is designed to improve the robust performance and velocity tracking accuracy. The designed rigid-body state observer allows a higher gain so that the bandwidth of the observer can increase, and the equivalent rigid-body velocity can be acquired more accurately. Notably, the high gain observer reduces the sensitivity to model uncertainties and exotic disturbances, especially near the resonant frequency. In addition, the observer combined with an adjustable damper can suppress the residual vibration and torque ripple simultaneously. The proposed method is compared experimentally with a PI method and two other rigid-body velocity methods, such as the conventional equivalent rigid-body observer method and the self-resonance cancellation method, to verify its advantages.
\end{abstract}

Keywords: modular joint; residual vibration; torque ripple; model uncertainties; external disturbances; robustness

\section{Introduction}

The modular joint with flexible Harmonic Drive (HD) is commonly used in collaborative robots due to its lightweight, high gear reduction ratio, and high power density [1]. However, its inherent resonance is easy to induce vibration if the command velocity changes abruptly, seriously affecting the system stability [2]. Moreover, due to the inaccurate dynamic models and impeded external disturbances, the accuracy of velocity control seriously degrades. Therefore, when the velocity servo signal varies quickly, it is essential to suppress the residual vibration on the modular joint and ensure its robustness in the presence of model uncertainties and external disturbances.

Up to now, various methods have been proposed for vibration suppression in modular joints [3]. Among the active vibration suppression methods, they can be roughly divided into trajectory planning and controller designing methods. Trajectory planning includes online and offline planning. The online planning designs an optimal trajectory to minimize vibrations, but significant computational resources are required [4]. On the contrary, offline planning enjoys less computational intensity, but it is generally applicable to repetitive motion. In addition, it is hard to apply to the scenarios that interact with the unknown 
environment [5-8]. The controller designing methods can be further divided into openloop control methods and closed-loop control methods. The input shaping is an openloop control method [9]. The key idea is to obtain the control input signal based on the reference command and vibrations. Then it modifies the input signal after taking the physical and vibrational properties of the modular joint into account to reduce vibrations. However, it has trouble withstanding system modeling error, which is sensitive to model uncertainties [10-12]. The closed-loop control methods have been credited in various applications as powerful tools to suppress vibrations, such as fuzzy logic and neural networks. The fuzzy logic controller is usually used to optimize the controller parameters to achieve vibration suppression. However, it is often hard to design parameters as it requires expert knowledge about the controlled system [13-15]. The neural network method is a fast approach for designing controllers without any prior knowledge about system dynamics. It uses advanced information about the input and output data relationships to compensate for the joint's nonlinearities, indirectly reducing the link side vibrations. Nevertheless, it is time-consuming and computationally complex. Furthermore, limited data information may lead to bad control performance [16-18].

In addition, there are other popular closed-loop control methods due to their good robust performance to model uncertainties and external disturbances. The quantitative feedback theory is based on the Nichols diagram in frequency domain to analyze systems' closed-loop performance and design the robust controller subsequently. It is robust to model uncertainties but requires heavy computations [19-21]. The Disturbance Observer (DOB) based method is robust to exotic disturbances. In this method, the model uncertainties are considered as disturbances, leading to poor tracking performance. Moreover, to reduce the bound of the robustness and improve the performance, the nominal model in DOB should be as accurate as possible [22-24]. The Active Disturbance Rejection Control (ADRC) requires little information about the physical plant. In the framework of ADRC, the Extended State Observer (ESO) is generally regarded as a fundamental part, which is used to estimate all measurements relating to system states and lumped disturbances [25]. However, the parameters tuning, especially the bandwidth of the ESO, are intricate and time-consuming [26,27].

In addition to the methods discussed above, there are some methods based on the idea of rigid-body velocity. The Self-Resonance Cancellation (SRC) method is a rigidbody velocity strategy to counteract the system resonance and anti-resonance to reduce vibrations [28]. It directly obtains the equivalent rigid-body velocity by the weighted sum of the modular joint's motor and load inertias without considering the closed-loop dynamics. So, it is sensitive to model uncertainties. An Equivalent Rigid-Body Observer (ERBO) method is also used to damp vibrations in [29]. Its observer acquires the equivalent rigid-body velocity, where the nominal model only includes the rigid-body dynamics without considering the flexible dynamics. The gain of its observer is affected by the resonant frequency, which increases the difficulty of gain tuning. Under the premise of system stability, the gain has to be small, resulting in the limited bandwidth of the observer and poor robustness to model uncertainties and external disturbances. In addition, it only works on the residual vibration while it has no effects on the torque ripple because of its phase adjuster, which reduces the velocity tracking accuracy during uniform motion.

This paper proposes a velocity control method to reduce the sensitivity to model uncertainties and external disturbances near the system resonant frequency, improving the robust stability and velocity tracking accuracy. The proposed method mainly includes a rigid-body state observer and an adjustable damper. The rigid-body state observer is designed the order of its nominal model as similar to the actual physical plant as possible, which lets the gain of this observer be pushed higher. Thereby, the high gain increases the bandwidth of the observer and improves the observation accuracy of the equivalent rigid-body velocity. Notably, the high gain can attenuate the adverse influences of the model uncertainties and exotic disturbances near the resonant frequency. In addition, when the feedback gain of the adjustable damper is tuned to a unique value, the proposed 
method can be equivalent to the SRC method but be more robust and stable. Ultimately, an adjustable damper combined with the high gain rigid-body state observer adds system damping to simultaneously reduce the residual vibration during acceleration/deceleration and torque ripple during low-speed uniform motion. In order to validate the advantages of this method, the experiments are compared with a PI method and two other rigid-body velocity methods, such as the ERBO method and the SRC method.

The contributions of this paper are:

1. The designed rigid-body state observer can compensate for model uncertainties, such as modeling errors and unmodeled system damping.

2. The designed rigid-body state observer combined with the adjustable damper can improve the robustness to model uncertainties and external disturbances.

3. The proposed method can simultaneously suppress the residual vibration induced by the system resonant frequency during acceleration/deceleration and torque ripple caused by the HD during low-speed uniform motion.

This paper is organized as follows. The modular joint is described and modelled in Section 2. The controller is designed in Section 3. The system identification, controller parameters analysis and robust stability analysis are arranged in Section 4. Next, the experiments and results are discussed in Section 5. Finally, Section 6 concludes this paper.

\section{System Description and Dynamic Modeling}

The modular joint integrates electronics and mechanical components, including a Permanent Magnet Synchronous Motor (PMSM), HD, shaft, bearings, dual encoders (motorside encoder and link-side encoder), torque sensor, and other components, as shown in Figure 1.

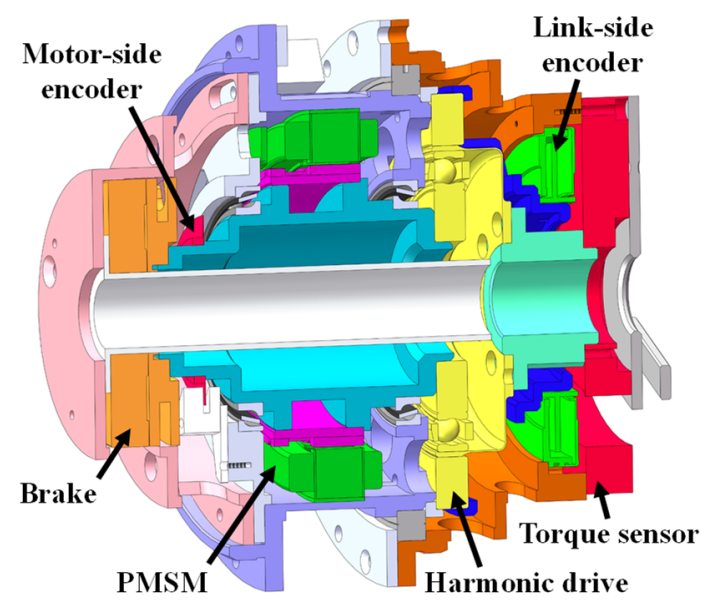

Figure 1. Section view diagram of the modular joint.

According to Figure 1, a two-inertia model is employed to describe the physical behavior in Figure 2a. The mathematical model diagram of the two-inertia model is shown in Figure $2 \mathrm{~b}$. Wherein, $\bar{J}_{m}, J_{l}, \bar{B}_{m}, B_{l}, \bar{\theta}_{m}, \theta_{l}$, and $n$ are the motor inertia, load inertia, motor viscous damping, load viscous damping, motor position, load position, and reduction ratio of the $\mathrm{HD}$, respectively. $\bar{\tau}_{m}, \tau_{j}$ indicate the input torque and joint torque. The drive chain (includes torque sensor and HD) is modelled as a linear spring $K$ and viscous damper $D$. Furthermore, $\bar{d}_{m}$ is the motor-side external disturbances, such as the modeling errors, nonlinear frictions, and unmodeled system damping. $d_{l}$ is the link-side external disturbances, such as the imprecise load inertia and unmodeled nonlinear environmental damping. $\bar{e}_{T E^{\prime}}$ is the transmission error of the HD, whose dominant frequency is twice related to the motor velocity [30]. 


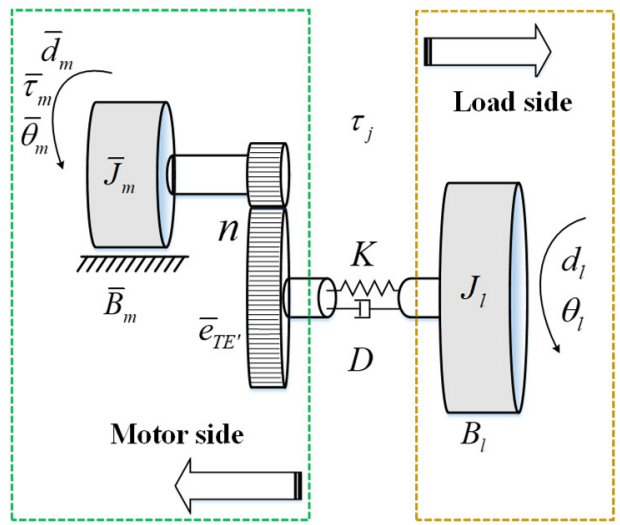

(a)

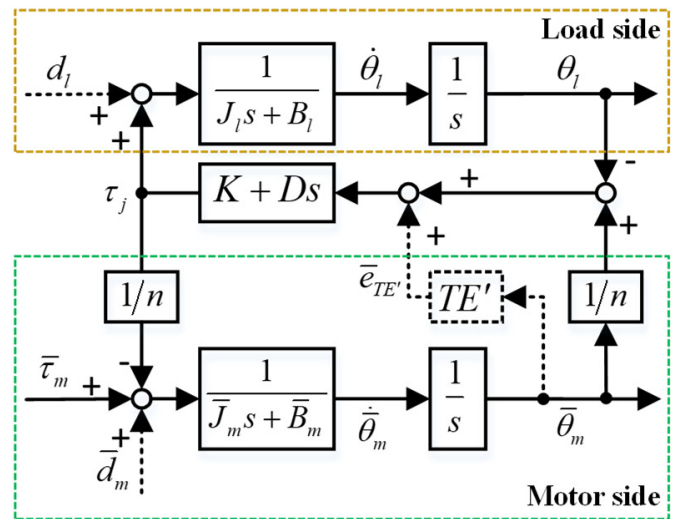

(b)

Figure 2. Two-inertia model diagrams of the modular joint. (a) Free body diagram. (b) Mathematical model diagram.

In general, the dynamic equations of the modular joint are expressed as a two-inertia model, as shown in the following [31]:

$$
\begin{gathered}
\bar{\tau}_{m}+\bar{d}_{m}-\frac{\tau_{j}}{n}=\bar{J}_{m} \ddot{\bar{\theta}}_{m}+\bar{B}_{m} \dot{\bar{\theta}}_{m} \\
\tau_{j}=K\left(\frac{\bar{\theta}_{m}}{n}-\theta_{l}+\frac{\bar{e}_{T E^{\prime}}}{n}\right)+D\left(\frac{\dot{\bar{\theta}}_{m}}{n}-\dot{\theta}_{l}+\frac{\dot{\bar{e}}_{T E^{\prime}}}{n}\right) \\
\tau_{j}+d_{l}=J_{l} \ddot{\theta}_{l}+B_{l} \dot{\theta}_{l}
\end{gathered}
$$

The parameters $\bar{\tau}_{m}, \bar{d}_{m}, \bar{\theta}_{m}, \bar{e}_{T E^{\prime}}, \bar{J}_{m}, \bar{B}_{m}$ are defined as $\tau_{m}=n \bar{\tau}_{m}, d_{m}=n \bar{d}_{m}, \theta_{m}=$ $\bar{\theta}_{m} / n, e_{T E}=\bar{e}_{T E^{\prime}} / n, J_{m}=n^{2} \bar{J}_{m}, B_{m}=n^{2} \bar{B}_{m}$. Therefore, the dynamic equations can be simplified to (2).

$$
\begin{gathered}
\tau_{m}+d_{m}-\tau_{j}=J_{m} \ddot{\theta}_{m}+B_{m} \dot{\theta}_{m} \\
\tau_{j}=K\left(\theta_{m}-\theta_{l}+e_{T E}\right)+D\left(\dot{\theta}_{m}-\dot{\theta}_{l}+\dot{e}_{T E}\right) \\
\tau_{j}+d_{l}=J_{l} \ddot{\theta}_{l}+B_{l} \dot{\theta}_{l}
\end{gathered}
$$

Moreover, to further simplify the modular joint's model, the external disturbances and the transmission error of HD are temporarily omitted. Consequently, the transfer function from input torque $\tau_{m}$ to motor velocity $\dot{\theta}_{m}$ and link velocity $\dot{\theta}_{l}$ in Laplace domain are given in (3) and (4), respectively.

$$
\begin{gathered}
P_{T M}(s)=\frac{\dot{\theta}_{m}}{\tau_{m}}=\left(\frac{1}{J_{m}+J_{l}} \frac{1}{s}\right) \cdot\left(\frac{s^{2}+\omega_{a}^{2}}{\omega_{a}^{2}} \frac{\omega_{r}^{2}}{s^{2}+\omega_{r}^{2}}\right) \\
P_{T L}(s)=\frac{\dot{\theta}_{l}}{\tau_{m}}=\left(\frac{1}{J_{m}+J_{l}} \frac{1}{s}\right) \cdot\left(\frac{\omega_{r}^{2}}{s^{2}+\omega_{r}^{2}}\right)
\end{gathered}
$$

where $\omega_{a}=\sqrt{K / J_{l}}, \omega_{r}=\sqrt{K\left(J_{m}+J_{l}\right) /\left(J_{m} J_{l}\right)}$.

\section{Controller Design}

In order to suppress the residual vibration during acceleration/deceleration and torque ripple during low-speed uniform motion, a robust velocity control method is proposed in this paper. The method mainly includes a rigid-body state observer, an adjustable damper, and a controller $C$, as shown in Figure 3. The error between link velocity and rigid-body velocity is additionally fed back beside the link velocity feedback. The motor and link velocity can be acquired from the motor-side and link-side encoders of the modular joint, which is estimated by the differentiation of the motor position and link position. The rigid-body velocity is a nominal velocity of the modular joint, which can be derived from (3) and (4) as $1 /\left(\left(J_{m}+J_{l}\right) s\right)$. However, in a two-inertia system, the 
rigid-body velocity cannot be measured directly by sensors. Therefore, how to acquire the rigid-body velocity is the fundamental issue that needs to be first considered.

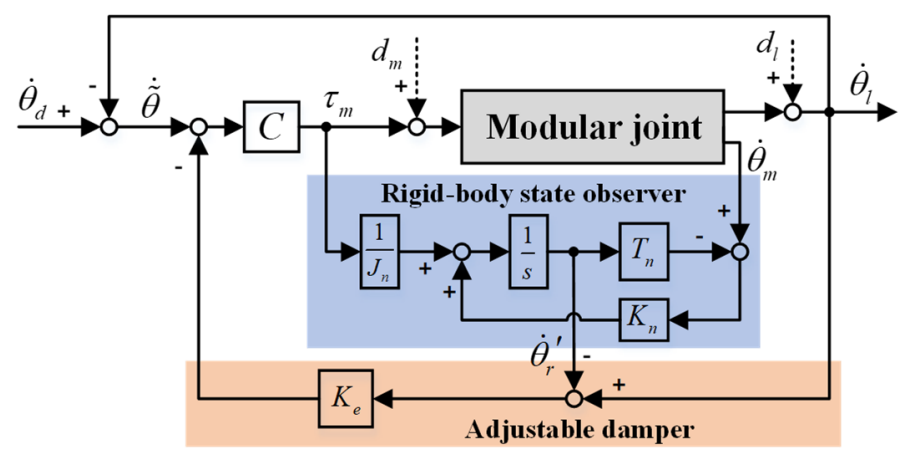

Figure 3. Block diagram of the proposed controller.

A rigid-body state observer is designed to obtain the equivalent rigid-body velocity. Then, based on the designed observer, an adjustable damper feeds back the error between link velocity and the observed equivalent rigid-body velocity to a controller $C$.

\subsection{Rigid-Body State Observer Design}

In earlier works, the modular joint system is described as a simplified two-inertia model in (2). Figure 4 shows the rigid-body state observer, where the nominal model $J_{n}$ is the sum of motor inertia and load inertia, expressed as $J_{n}=J_{m}+J_{l}$. Firstly, we assume that there is no error between the physical model $J$ and the nominal model $J_{n}$, so $J=J_{n}$.

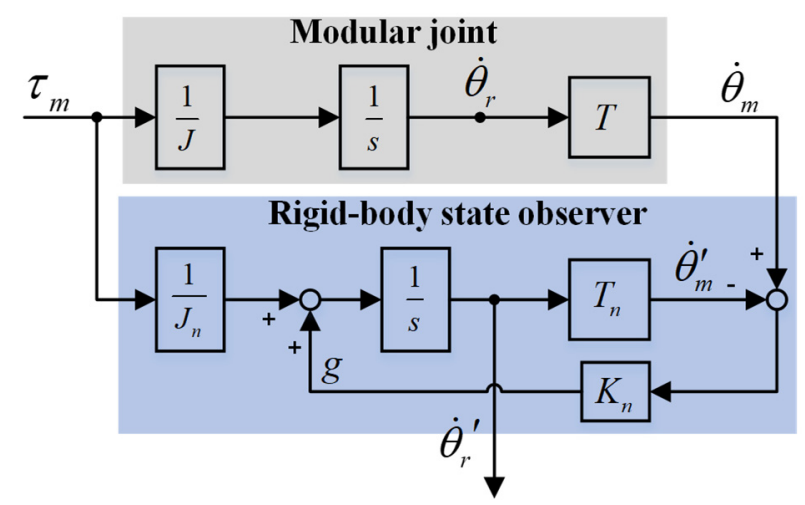

Figure 4. Block diagram of the rigid-body state observer.

The closed-loop feedback value $g$ of this observer, marked in Figure 4, can be expressed as:

$$
g=\tau_{m}\left(\frac{T}{J_{S}}-\frac{T_{n}}{J_{n} S}\right) K_{n}=\frac{\tau_{m}}{J_{n} S}\left(T-T_{n}\right) K_{n}
$$

The transfer function $R$ from the actual rigid-body velocity $\dot{\theta}_{r}$ to the observed rigidbody velocity $\dot{\theta}_{r}{ }^{\prime}$ is given as:

$$
R=\frac{\dot{\theta}_{r}{ }^{\prime}}{\dot{\theta}_{r}}=\frac{\frac{J}{J_{n}} s+K_{n} T}{s+K_{n} T_{n}}=\frac{s+K_{n} T}{s+K_{n} T_{n}}
$$

The nominal model $T_{n}$ is designed as $T_{n}=T$ in the rigid-body state observer. In this case, the closed-loop feedback value $g$ in (5) becomes $g=0$, which means the gain $K_{n}$ can be infinite theoretically. Similarly, the observed rigid-body velocity always equals the actual rigid-body velocity as $R=1$ with any gain $K_{n}$ in (6). Under the premise of system stability 
and $R=1$, the model uncertainties and external disturbances can be effectively cancelled with a sufficiently high gain $K_{n}$.

In order to avoid the identification of $T_{n}, T_{n}$ is designed to be unity in [29], where the nominal model of its observer only contains the rigid-body dynamics. In this observer, the closed-loop feedback value $g$ in (5) becomes:

$$
g=\frac{\tau_{m}}{J_{n} s}\left(T-T_{n}\right) K_{n}=\frac{\tau_{m}}{J_{n} s}(T-1) K_{n}
$$

The transfer function $R$ in (6) becomes:

$$
R=\frac{\dot{\theta}_{r}^{\prime}}{\dot{\theta}_{r}}=\frac{s+K_{n} T}{s+K_{n} T_{n}}=\frac{s+K_{n} T}{s+K_{n}}
$$

From (7), the gain $K_{n}$ is affected by the resonant frequency, which leads to difficulty in $K_{n}$ tuning. Particularly, the system may become unstable during high acceleration and deceleration. Thus, the gain $K_{n}$ has to be small enough to ensure the system stability, which unfortunately causes the poor robustness to model uncertainties and exotic disturbances. In addition, the observed rigid-body velocity in (8) is also influenced by the resonant frequency, which reduces the observation accuracy of the equivalent rigid-body velocity. This further weakens the vibration suppression performance in the outer loop.

Considering the robust stability of the system to model uncertainties and exotic disturbances, the nominal model $T_{n}$ is designed as:

$$
T_{n}=T=\frac{s^{2}+\omega_{a}^{2}}{s^{2}+\omega_{r}^{2}} \frac{\omega_{r}^{2}}{\omega_{a}^{2}}
$$

Actually, there are unavoidable model uncertainties on the physical plants regarding $J$ and $T$. The rigid-body state observer based on $H_{\infty}$ norm optimization can strictly guarantee robust stability to the model uncertainties [23]. Suppose that the model uncertainties can be treated as a multiplicative perturbation in (10), where the perturbation $\Delta(s)$ and $\Omega(s)$ are assumed to be stable. So, $J$ and $T$ are represented as:

$$
\begin{gathered}
J(s)=J_{n}(s)[I+\Delta(s)] \\
T(s)=T_{n}(s)[I+\Omega(s)]
\end{gathered}
$$

The rigid-body state observer is robust stable if:

$$
\begin{aligned}
& \bar{\sigma}_{1}\left(\Delta(j \omega) \cdot K_{n}(j \omega)\right)<1 \\
& \bar{\sigma}_{2}\left(\Omega(j \omega) \cdot K_{n}(j \omega)\right)<1
\end{aligned}, \forall \omega
$$

where $\bar{\sigma}_{1}(\cdot)$ and $\bar{\sigma}_{2}(\cdot)$ represent the maximum of singular value.

However, the rigid-body state observer is only a part of the overall control system. Robust stability to model uncertainties should be considered with the outer loop. To comprehensively analyze the model uncertainties, define $\gamma(s)$ as the total model perturbation, expressed as:

$$
\gamma(s)=\Delta(s)+\Omega(s)
$$

The transfer function from the output of the total model perturbation $\gamma(s)$ to its input is:

$$
\zeta(s)=\frac{C K_{e} K_{n} P_{T M}-C\left(K_{e}+1\right)\left(s+K_{n} T_{n}\right) P_{T L}}{C\left(K_{e}+1\right)\left(s+K_{n} T_{n}\right) P_{T L}-C K_{e}\left(J_{n}^{-1}+K_{n} P_{T M}\right)-\left(s+K_{n} T_{n}\right)}
$$

Suppose $\gamma(s)$ is bounded by the upper limit function $\lambda_{U}(s)$ as:

$$
|\gamma(j \omega)| \leq\left|\lambda_{U}(j \omega)\right|, \forall \omega .
$$


Then, satisfaction of the following condition guarantees the robust stability of the whole closed-loop system:

$$
\left\|\lambda_{U}(s) \cdot \frac{C K_{e} K_{n} P_{T M}-C\left(K_{e}+1\right)\left(s+K_{n} T_{n}\right) P_{T L}}{C\left(K_{e}+1\right)\left(s+K_{n} T_{n}\right) P_{T L}-C K_{e}\left(J_{n}-1+K_{n} P_{T M}\right)-\left(s+K_{n} T_{n}\right)}\right\|_{\infty}<1
$$

\subsection{Adjustable Damper Design}

During the adjustable damper design, some assumptions are prepared for simplifying the analysis of the closed-loop performance of the controller. The physical plants $J$ and $T$ are assumed to equal the nominal models $J_{n}$ and $T_{n}$ without model uncertainties, shown as (16).

$$
\begin{aligned}
J(s) & =J_{n}(s) \\
T(s) & =T_{n}(s)
\end{aligned}
$$

Under these assumptions, the observed velocity $\dot{\theta}_{r}^{\prime}$ in the rigid-body state observer is equal to the actual velocity $\dot{\theta}_{r}$, directly calculated in (17) without the control parameter $K_{n}$.

$$
\frac{\dot{\theta}_{r}{ }^{\prime}}{\tau_{m}}=\frac{\dot{\theta}_{r}}{\tau_{m}}=\left(\frac{1}{J_{m}+J_{l}} \frac{1}{s}\right)=\frac{1}{J_{n} \mathcal{S}}
$$

Therefore, with the assumptions in (16), the adjustable damper can be designed separately without considering the control parameter $K_{n}$. Define the velocity error $\dot{\widetilde{\theta}}$ between the desire velocity $\dot{\theta}_{d}$ and link velocity $\dot{\theta}_{l}$ as $\dot{\widetilde{\theta}}=\dot{\theta}_{d}-\dot{\theta}_{l}$, marked in Figure 3 . Thus, the open-loop transfer function from $\dot{\widetilde{\theta}}$ to $\dot{\theta}_{l}$ is given as:

$$
P_{\text {open }}(s)=\frac{\dot{\theta}_{l}}{\dot{\tilde{\theta}}}=\frac{C}{J_{n} s} \frac{\omega_{r}^{2}}{s^{2}+\omega_{r}^{2}}
$$

The closed-loop transfer function from $\dot{\widetilde{\theta}}$ to $\dot{\theta}_{l}$ is given as:

$$
P_{\text {close }}(s)=\frac{\dot{\theta}_{l}}{\dot{\tilde{\theta}}}=\frac{C}{J_{n} s} \frac{\omega_{r}^{2}}{s^{2}-K_{e} C s+\omega_{r}^{2}}
$$

Compared (19) with (18), the closed-loop transfer function adds a damping term- $K_{e} C s$ to the modular joint system. As the gain $K_{e}$ is negative, the system damping is increased. When the modular joint moves during acceleration and deceleration, the added damping term can suppress the residual vibration. Meanwhile, the increased system damping also can eliminate the torque ripple during the low-speed uniform motion to advance the velocity tracking accuracy.

In addition, the modular joint also experiences external disturbances, including motorside exotic disturbances $d_{m}$ and link-side exotic disturbances $d_{l}$, as shown in Figure 3 .

The sensitivity function $D M$ from $d_{m}$ to $\dot{\theta}_{l}$ is:

$$
D M=\frac{\dot{\theta}_{l}}{d_{m}}=\frac{\omega_{r}^{2} s+\left(C K_{e}+K_{n} T_{n}\right) \omega_{r}^{2}}{s^{4}+a s^{3}+b s^{2}+c s+d}
$$

where $a=C K_{e}+K_{n} T_{n}$,

$b=\omega_{r}{ }^{2}\left(\omega_{a}{ }^{2}-C K_{e} K_{n}\right) / \omega_{a}{ }^{2}$,

$c=\left(C+2 C K_{e}+K_{n} T_{n}\right) \omega_{r}{ }^{2}$,

$d=C K_{n}\left(T_{n}+T_{n} K_{e}-K_{e}\right) \omega_{r}{ }^{2}$. 
The sensitivity function $D L$ from $d_{l}$ to $\dot{\theta}_{l}$ is:

$$
D L=\frac{\dot{\theta}_{l}}{d_{l}}=\frac{s^{4}+a s^{3}+\omega_{r}^{2} s^{2}+\left(C K_{e}+K_{n} T_{n}\right) \omega_{r}^{2} s}{s^{4}+a s^{3}+b s^{2}+c s+d}
$$

where $a=C K_{e}+K_{n} T_{n}$,

$b=\omega_{r}^{2}\left(\omega_{a}^{2}-C K_{e} K_{n}\right) / \omega_{a}^{2}$,

$c=\left(C+2 C K_{e}+K_{n} T_{n}\right) \omega_{r}^{2}$,

$d=C K_{n}\left(T_{n}+T_{n} K_{e}-K_{e}\right) \omega_{r}^{2}$.

These external disturbances unavoidably degrade the velocity control accuracy, especially when the tracking velocity changes sharply. Therefore, the robustness to external disturbances near the resonant frequency should be considered.

\section{Controller Analysis}

To analyze the proposed controller's robustness performance and velocity tracking accuracy, the dynamic parameters of the controlled object-modular joint need to be identified first. The modular joint installed some loads is publicized in Figure 5. The installed loads compose the modular joint's load inertia.

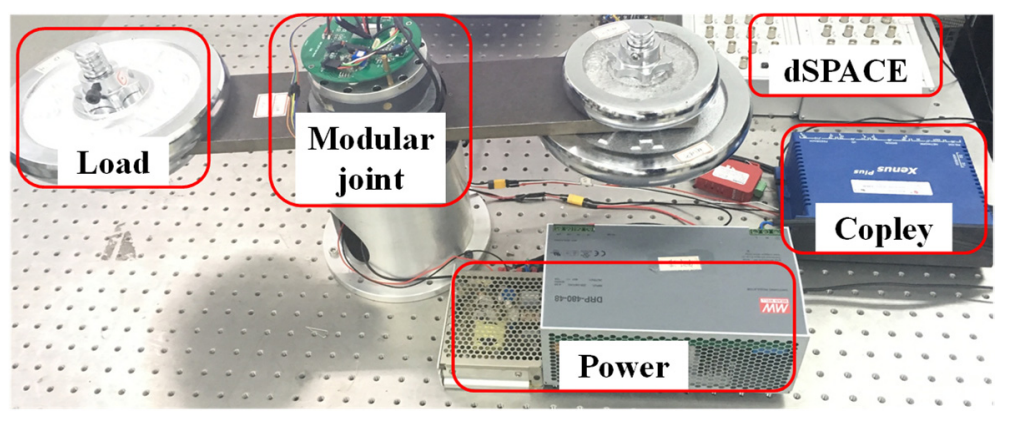

Figure 5. Physical diagram of the experimental platform.

\subsection{System Identification}

The experimental platform includes a modular joint, a $24 \mathrm{~V}$ power, a Copley driver, a dSPACE real-time control system, several loads, and a MATLAB data processing system, as shown in Figure 5.

The sampling frequency is set as $1 \mathrm{kHz}$. The gear ratio $n$ of $\mathrm{HD}$ is 160 . The torque constant of motor is $0.17 \mathrm{~N} \cdot \mathrm{m} / \mathrm{A}$. The input saturation current is set to be $10 \mathrm{~A}$. The maximum allowable desire velocity is $1.64 \mathrm{rad} / \mathrm{s}$. The motor velocity and link velocity are obtained by the differentiation of the motor position and link position, which are measured by the motor-side encoder and link-side encoder, respectively.

A swept sine signal from $0.5 \mathrm{~Hz}$ to $60 \mathrm{~Hz}$ with a load inertia $J_{l}=2.26 \mathrm{~kg} \cdot \mathrm{m}^{2}$ is applied to identify the modular joint's open-loop dynamic characteristics. According to system identification results, the modular joint's frequency responses from input torque to motor velocity and link velocity are displayed in Figure 6a,b, respectively.

The blue dash-dot lines are the results of the system identification experiments. The red thick solid lines are fitted frequency responses of the established two-inertia model. Whereas, the actual modular joint's dynamic model is subjected to several nonlinear and time-varying factors, such as the nonlinear frictions and viscous damping effects in the motor-side and transmission part, and the varying efficiency of the HD (60-75\% depending on ratio, velocity, and lubricant). In particular, the stiffness and damping of the HD are related to the motion velocity. Therefore, the established two-inertia model cannot accurately fit the experimental results, especially the phase [32]. However, the system's anti-resonant frequency and resonant frequency can be well fitted at $19 \mathrm{~Hz}$ and $21.8 \mathrm{~Hz}$, proving that the two-inertia model can reflect the joint's dynamic characteristics. The dynamic parameters of the modular joint can be identified in Table 1. 

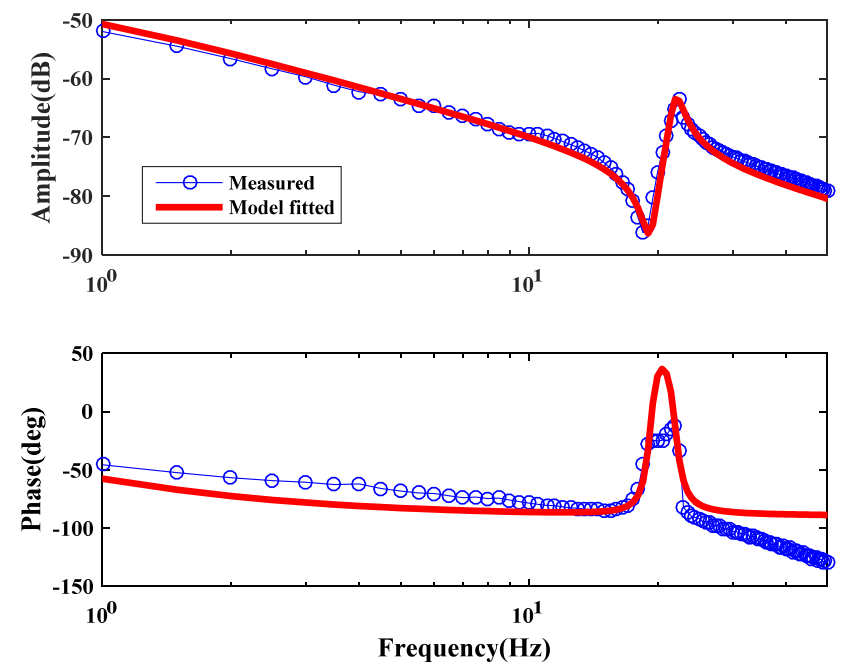

(a)
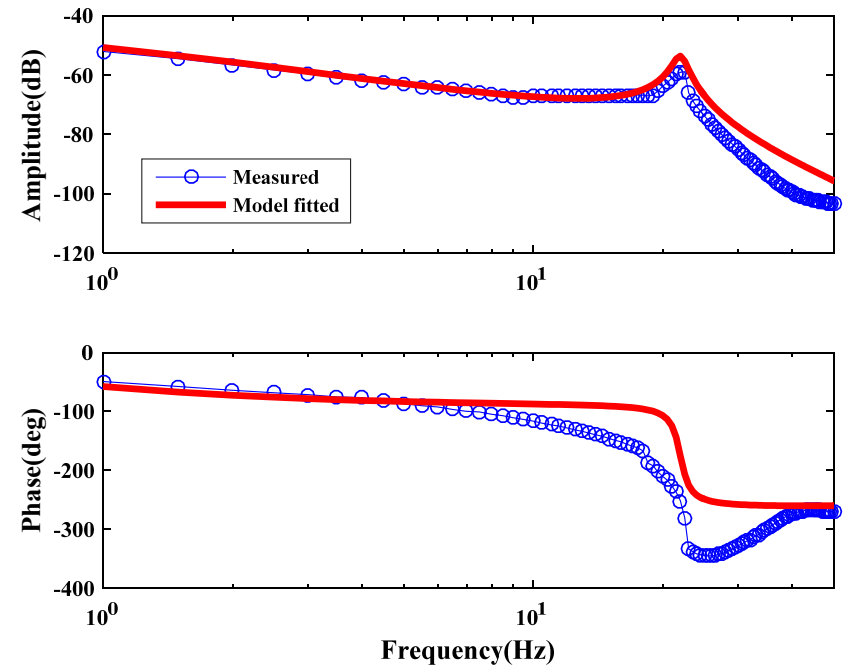

(b)

Figure 6. Modular joint frequency responses. (a) From input torque to motor velocity. (b) From input torque to link velocity.

Table 1. Identified modular joint's dynamic parameters.

\begin{tabular}{cccc}
\hline Symbol & Name & Value & Unit \\
\hline$J_{m}$ & Motor inertia & 7.34 & $\mathrm{~kg} \cdot \mathrm{m}^{2}$ \\
$B_{m}$ & Motor viscous & 33.28 & $\mathrm{~N} \cdot \mathrm{m} \cdot \mathrm{s} / \mathrm{rad}$ \\
$J_{l}$ & damping & 2.26 & $\mathrm{~kg} \cdot \mathrm{m}^{2}$ \\
$B_{l}$ & Load inertia & 5.00 & $\mathrm{~N} \cdot \mathrm{m} \cdot \mathrm{s} / \mathrm{rad}$ \\
$K$ & dad viscous & $32,500.00$ & $\mathrm{~N} \cdot \mathrm{m} / \mathrm{rad}$ \\
$D$ & Joint stiffness & 10.00 & $\mathrm{~N} \cdot \mathrm{m} \cdot \mathrm{s} / \mathrm{rad}$ \\
\hline
\end{tabular}

\subsection{Controller Parameters Analysis}

To meet the rapid response of the modular joint, it is necessary first to ensure the velocity response performance. Based on the Integral of Squared Error (ISE) criterion [33], the controller $C$ is designed as a proportional and integral controller, tuned as $K_{p}=160$ and $K_{i}=1200$. 
Since the rigid-body state observer and the adjustable damper are designed separately, the control parameter $K_{n}$ of the observer and $K_{e}$ of the adjustable damper can also be designed separately. As the rigid-body state observer is shown in Figure 4, the transfer function from motor velocity $\dot{\theta}_{m}$ to the observed velocity $\dot{\theta}_{m}{ }^{\prime}$ can be expressed in (22).

$$
\frac{\dot{\theta}_{m}{ }^{\prime}}{\dot{\theta}_{m}}=\frac{K_{n} \omega_{r}{ }^{2} s^{2}+K_{n} \omega_{r}{ }^{2} \omega_{a}{ }^{2}}{\omega_{a}{ }^{2} s^{3}+K_{n} \omega_{r}{ }^{2} s^{2}+\omega_{r}{ }^{2} \omega_{a}{ }^{2} s+K_{n} \omega_{r}{ }^{2} \omega_{a}{ }^{2}}
$$

With the identified modular joint's dynamic parameters listed in Table 1, the bode plots from motor velocity to the observed velocity are obtained under the different control parameter $K_{n}$ in Figure 7. With the gain $K_{n}$ increasing from 100 to 1000, the bandwidth of the rigid-body state observer increases.

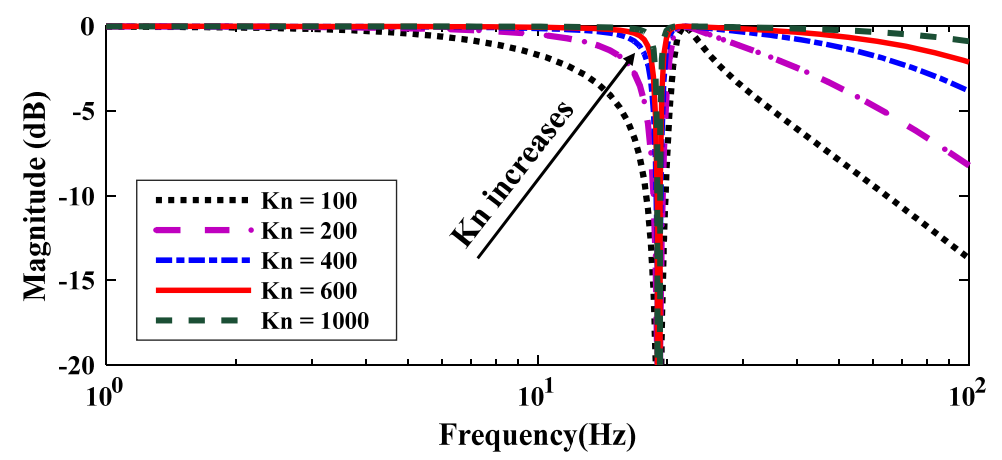

Figure 7. Bode plots from motor velocity to the observed velocity with different parameter $K_{n}$.

In addition, as the value of the control parameter $K_{e}$ changes, the open-loop and closed-loop frequency responses from velocity error $\dot{\widetilde{\theta}}$ to link velocity $\dot{\theta}_{l}$ are shown in Figure 8 . With the absolute value of the gain $K_{e}$ increasing, the vibration magnitude at resonant frequency decreases accordingly. When the added damping is too small, such as $K_{e}=-0.1$, the vibrations cannot be suppressed effectively because of the limited damping added. When the added damping is too large, such as $K_{e}=-2$, the system response slows down, meanwhile low-frequency uncertainty increases.

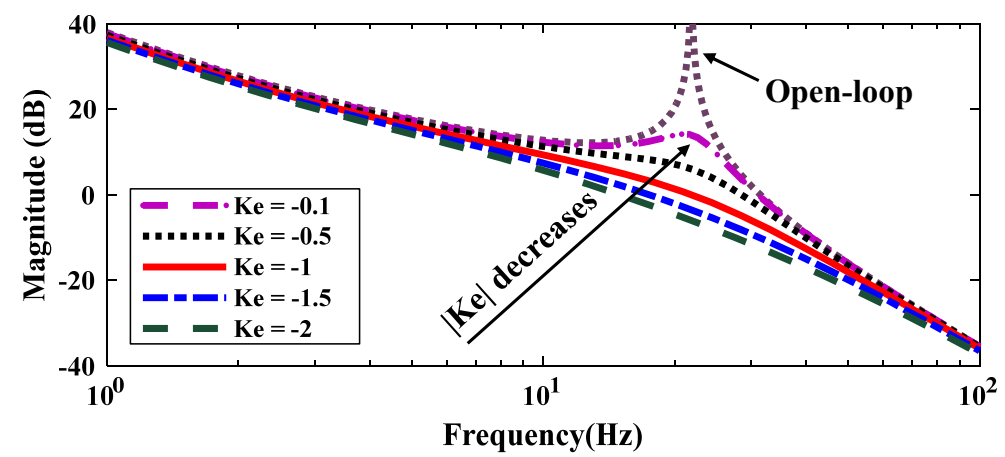

Figure 8. Bode plots from velocity error to link velocity with different parameter $K_{e}$.

The most conservative condition to ensure the controlled system is stable with little vibration is to let the amplitude ratio of the closed-loop frequency response in (19) be $0 \mathrm{~dB}$ at the resonant frequency, as expressed in (23).

$$
\left\{\begin{array}{c}
20 \log _{10}\left(P_{\text {close }}\left(j \omega_{r}\right)\right)=0 d B \\
P_{\text {close }}\left(j \omega_{r}\right)=\left.\frac{\dot{\theta}_{l}}{\dot{\tilde{\theta}}}\right|_{s=j \omega_{r}}=1
\end{array}\right.
$$


The theoretical value of $K_{e}$ from (23) can be calculated as -1 . Considering the model uncertainties and nonlinear damping effects, the parameter $K_{e}$ needs to be adjusted in a small range around -1 in actual experiments. Most importantly, in order to meet the robust stability condition of the whole closed-loop system as designed in (15), the controller's parameters of the proposed method are designed in Table 2.

Table 2. Controller parameters of the proposed method.

\begin{tabular}{ccc}
\hline Symbol & Name & Value \\
\hline$K_{p}$ & Proportional gain of $C$ & 160 \\
$K_{i}$ & Integral gain of $C$ & 1200 \\
$K_{n}$ & Gain of rigid-body state & 600 \\
$K_{e}$ & observer & -1.2 \\
\hline
\end{tabular}

\subsection{Robust Stability Analysis}

Further to analyze the robust performance of the rigid-body state observer, the model perturbations $\Delta(s)$ of $J_{n}$ and $\Omega(s)$ of $T_{n}$ are separately considered. In this paper, the model uncertainties mainly refer to the modeling errors, unmodeled system damping, system identification errors, even the imprecise motor and load inertia.

$T_{\Delta}(s)$ is the transfer function from the output of the model perturbation $\Delta(s)$ to its input. $T_{\Omega}(s)$ is the transfer function from the output of the model perturbation $\Omega(s)$ to its input. With the proposed method, the transfer function $T_{\Delta}(s)$ and $T_{\Omega}(s)$ can be expressed as (24) and (25), respectively.

$$
\begin{aligned}
& T_{\Delta}(s)=\frac{s}{s+K_{n} T_{n}(s)} \\
& T_{\Omega}(s)=\frac{K_{n} T_{n}(s)}{s+K_{n} T_{n}(s)}
\end{aligned}
$$

For a fair comparison, optimal controller parameters are employed in the ERBO method, SRC method, and the proposed method, respectively. The bode plots of the transfer function $T_{\Delta}(s)$ and $T_{\Omega}(s)$ with these three methods are compared in Figure $9 a, b$, respectively.

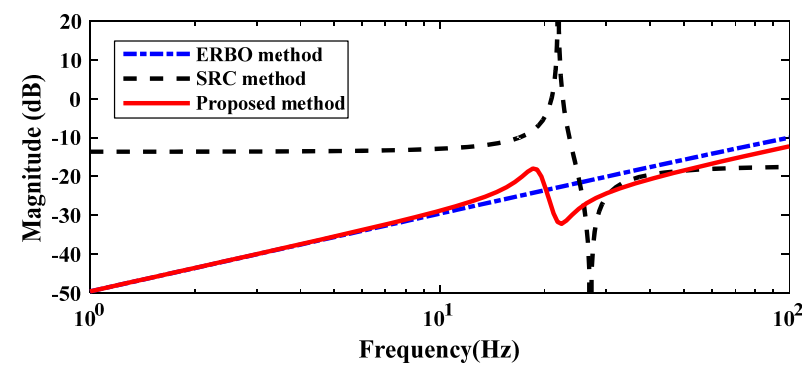

(a)

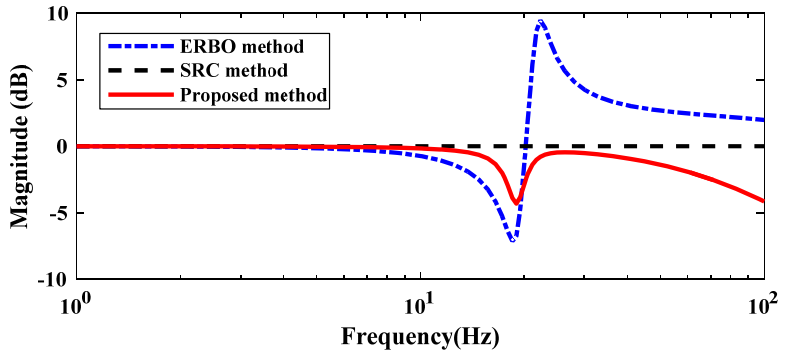

(b)

Figure 9. Bode plots of the model uncertainties. (a) Model uncertainties of $J_{n}$. (b) Model uncertainties of $T_{n}$. 
From Figure 9a, the SRC method is susceptible to the model uncertainties of $J_{n}$, especially at the low-frequency band and anti-resonant frequency. Compared with the ERBO method, the proposed method reduces the sensitivity to the model uncertainties near the resonant frequency, increasing system robustness. Although it improves the sensitivity near anti-resonant frequency, the characteristic of anti-resonance makes the system unresponsive, which means this introduced shortcoming is not that serious.

From Figure 9b, the ERBO method is susceptible to the model uncertainties of $T_{n}$, especially near the resonant frequency and high frequency. The SRC method is derived from the model $J_{n}$ in an open-loop, which is little related to the model uncertainties of $T_{n}$. Compared with the ERBO and SRC methods, the proposed method significantly reduces the sensitivity to model uncertainties near the resonant frequency and high frequency.

In addition, robust performance to model uncertainties can be verified with a specific physical indicator. Under the premise of ensuring that the dynamic parameters in Table 1 and controller parameters in Table 2 remain unchanged, the total model perturbation $\gamma(s)$ is simulated by changing the load inertia. The closed-loop transfer function from desire velocity $\dot{\theta}_{d}$ to link velocity $\dot{\theta}_{l}$ is expressed in (26).

$$
\frac{\dot{\theta}_{l}}{\dot{\theta}_{d}}=\frac{C \omega_{r}^{2}}{J_{n} s\left(s^{2}-K_{e} C s+\omega_{r}^{2}\right)+C \omega_{r}^{2}}
$$

As the load inertia changes within $J_{l} \pm 30 \%$, the frequency response of the closed-loop transfer function in (26) is shown in Figure 10.

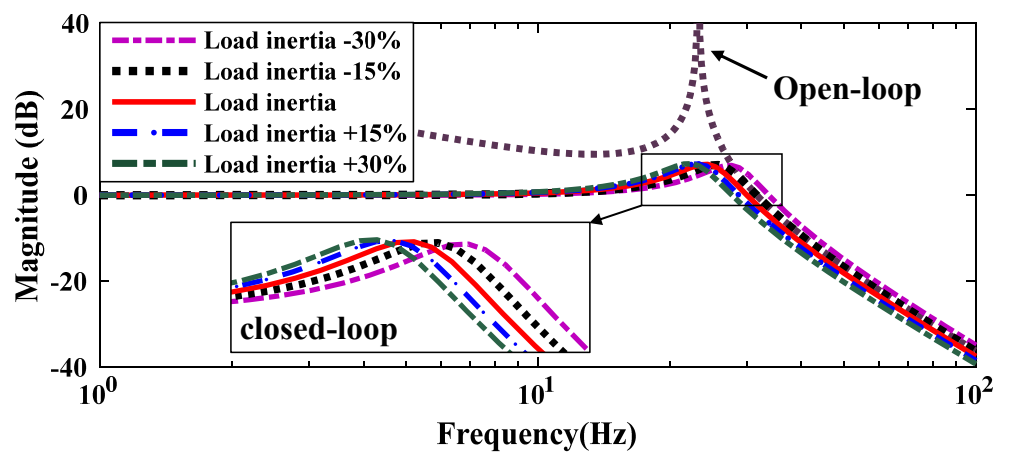

Figure 10. Bode plots from desire velocity to link velocity with different load inertia.

Compared to the open-loop frequency response, the oscillation magnitude of closedloop near resonant frequency can be damped effectively. With the load inertia increasing, the system resonant frequency decreases accordingly. It is obvious that the closed-loop system is robust and stable to the load inertia changing.

What's more, the modular joint is also influenced by the motor-side and link-side external disturbances. In order to analyze the robustness to external disturbances of the proposed method, Figure $11 \mathrm{a}, \mathrm{b}$, respectively, show bode plots of the sensitivity functions $D M$ and $D L$, expressed in (20) and (21). The ERBO method and SRC method are also compared and discussed following.

In particular, according to the feedback signals, when the feedback gain $K_{e}$ of the adjustable damper is tuned to -1 , the proposed method can be equivalent to the SRC method. Fortunately, the proposed method contains a closed-loop rigid-body state observer and an adjustable damper, which ensures the system robust stability performance. Justifiably, from the bode plots of these three methods, the SRC method behaves with the worst robustness on the low-frequency domain. The ERBO method behaves with the worst robustness near the resonant frequency. Compared with the SRC method and ERBO method, the proposed method is the most robust to the external disturbances, especially near the resonant frequency, which means its ability to work against exotic disturbances during high acceleration and deceleration is stronger than the other two methods. 


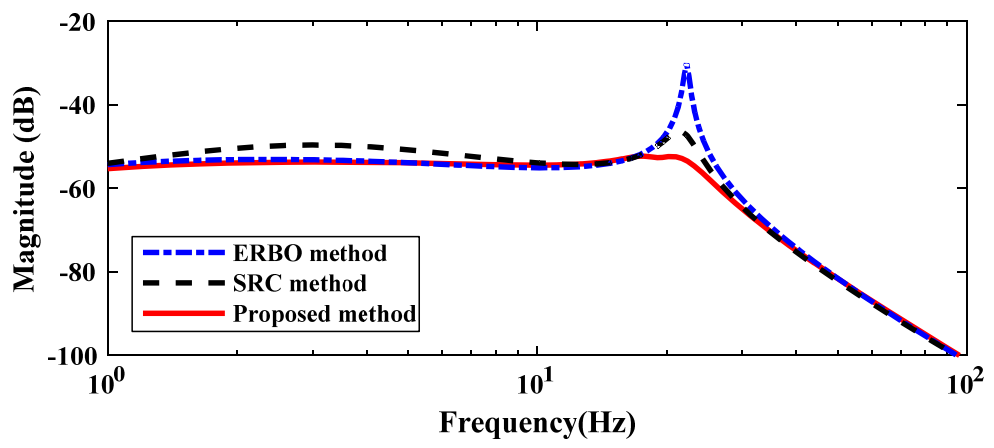

(a)

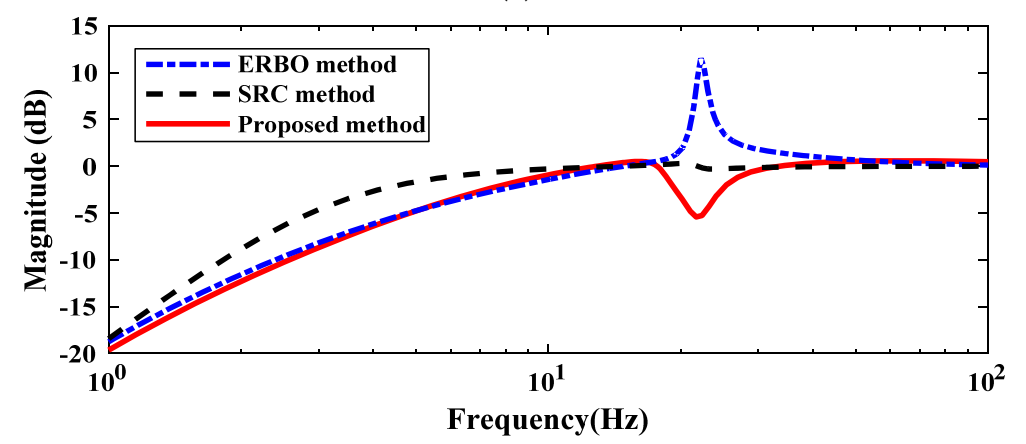

(b)

Figure 11. Bode plots of the external disturbances sensitivity function. (a) Motor-side disturbances $d_{m}$. (b) Link-side disturbances $d_{l}$.

\section{Experiments}

In this section, some experiments are carried out to verify the vibration suppression effects and the robust performance of the proposed method. The experimental platform has been exhibited in Figure 5.

\subsection{Performance of Vibration Suppression}

In experiments, the desire velocity is a step waveform to simulate the accelerated motion so that its spectrum overlaps the system resonant frequency. During this motion, the residual vibration is easy to be induced by the system resonant frequency. Therefore, a desire velocity of $0.33 \mathrm{rad} / \mathrm{s}$ is stepped from 0 at $0.1 \mathrm{~s}$, then keeps uniform motion for $1.4 \mathrm{~s}$. In $1.5 \mathrm{~s}$, the desire velocity stepped from 0.33 to $0.85 \mathrm{rad} / \mathrm{s}$ instantaneously and then maintained uniform motion for $1 \mathrm{~s}$. Figure 12a shows entire velocity tracking process, and the corresponding input current response is shown in Figure $12 \mathrm{~b}$.

Under the premise of unsaturated input current, the proposed method is compared with the PI method, ERBO method, and SRC method under their respective optimal controller parameters. In order to intuitively compare and quantify the vibration suppression effects, the average oscillation of the PI method is used as the benchmark, and the other average oscillations of the ERBO method, SRC method, and proposed method can be expressed as a percentage, respectively.

From Figure 12a, during the transient phase at $0.33 \mathrm{rad} / \mathrm{s}$, the percentage reduction in residual vibration with the ERBO method, SRC method, and proposed method is $45.7 \%$, $62.9 \%$, and $70.2 \%$, respectively. During the uniform motion phase at $0.33 \mathrm{rad} / \mathrm{s}$, the modular joint is influenced by a torque ripple, which is twice the tracking velocity frequency. The percentage reduction in the torque ripple with the ERBO method, SRC method, and proposed method is $14.9 \%, 70.2 \%$, and $76.3 \%$, respectively. Next, during the transient response from 0.33 to $0.85 \mathrm{rad} / \mathrm{s}$, the residual vibration with the ERBO method, SRC method, and proposed method reduces $65.8 \%, 74.0 \%$, and $86.3 \%$, respectively. Whereas there is no effect on the torque ripple during the uniform motion at $0.85 \mathrm{rad} / \mathrm{s}$ because the twice frequency of $0.85 \mathrm{rad} / \mathrm{s}$ is larger than the system resonant frequency, which is 
submerged in noises. Due to the better stability of the proposed method, its controller parameters can be tuned higher, which leads to a larger overshoot of its transient response. Fortunately, its vibration suppression ability will be stronger, which lets the residual vibration attenuate faster. Meanwhile, the torque ripple becomes much smaller than the other two methods.

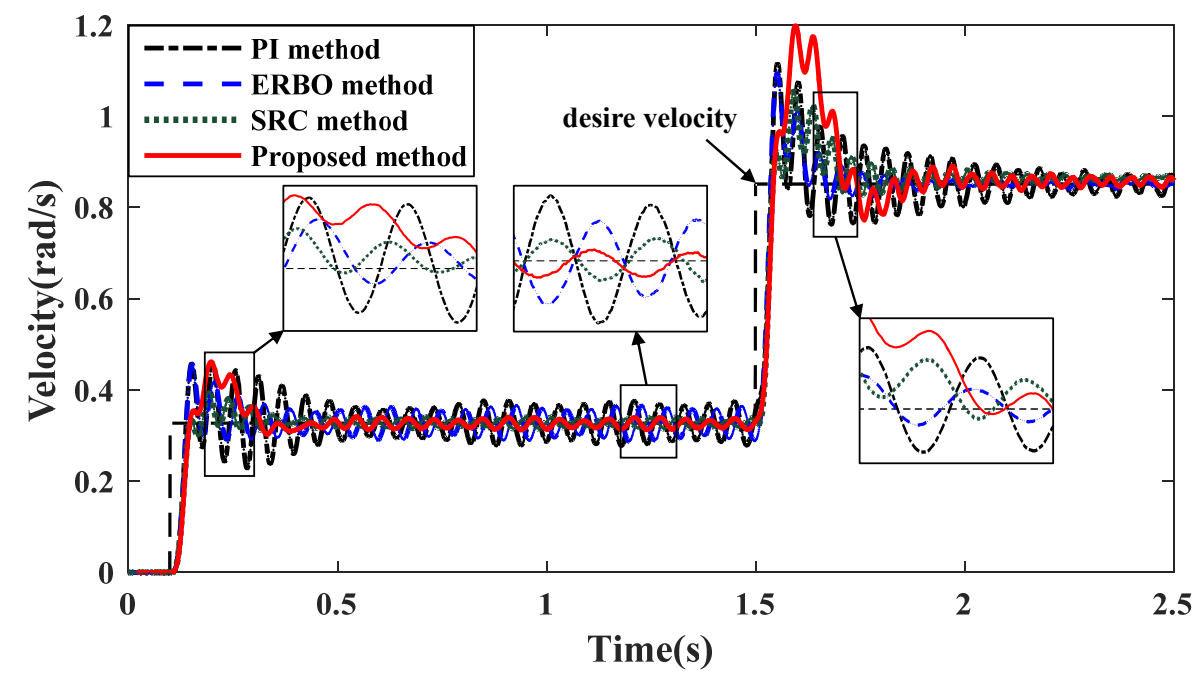

(a)

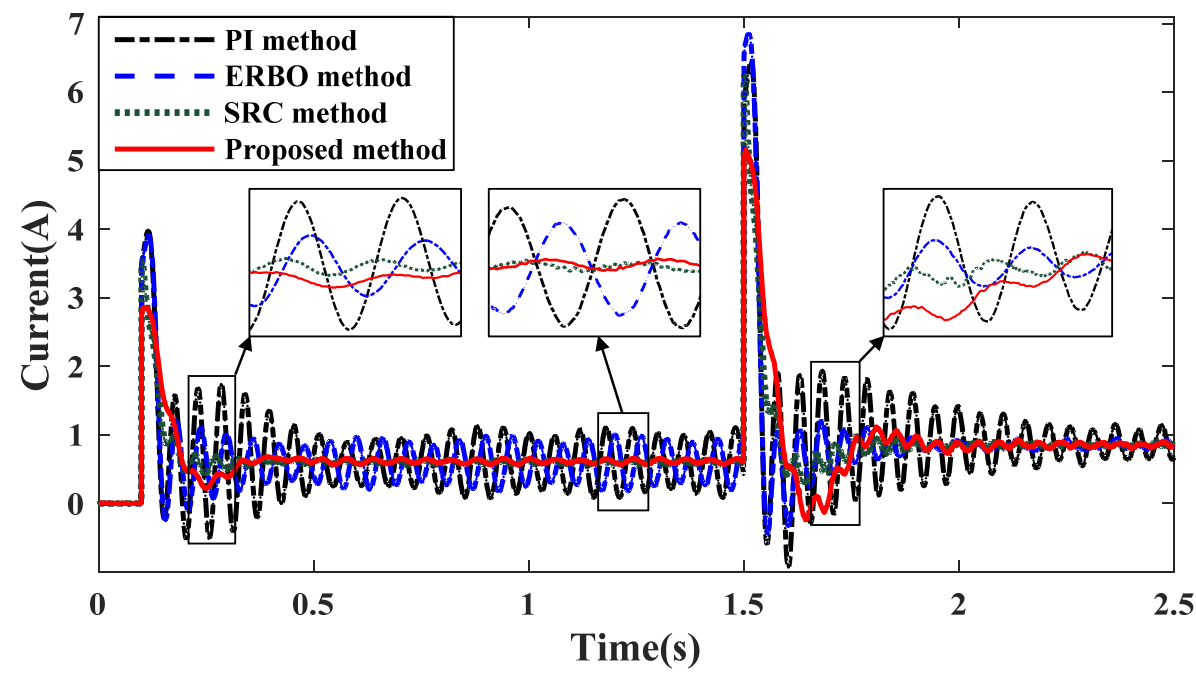

(b)

Figure 12. Vibration suppression experiments of velocity tracking. (a) Link velocity response. (b) Input current response.

As Figure 12b shows, there is around 0.6 A offset of the input current during the uniform motion to overcome the joint's frictions. Compared to the PI method, the residual vibration at the transient response of $0.33 \mathrm{rad} / \mathrm{s}$ with the ERBO method, SRC method, and proposed method reduces $51.1 \%, 86.1 \%$, and $93.2 \%$, respectively. The torque ripple at $0.33 \mathrm{rad} / \mathrm{s}$ reduces $23.0 \%, 91.2 \%$, and $91.2 \%$, respectively. Similarly, the residual vibration during the transient response from 0.33 to $0.85 \mathrm{rad} / \mathrm{s}$ reduces $56.6 \%, 70.6 \%$, and $80.1 \%$, respectively. Notably, the input current of the proposed method is smaller than the other two methods at the transient response. It means that the proposed method is energy-saving while ensuring the vibration suppression effects.

To avoid losing generality, the vibration suppression effects at other desire velocities, such as $0.20 \mathrm{rad} / \mathrm{s}, 0.46 \mathrm{rad} / \mathrm{s}, 0.66 \mathrm{rad} / \mathrm{s}$, are also verified in experiments. The percentage 
of reduction oscillations with the ERBO method, SRC method, and proposed method with respect to the PI method are summarized in Table 3.

Table 3. Reduction of oscillations compared with the Proportional-Integral method.

\begin{tabular}{cccccc}
\hline \multirow{2}{*}{$\begin{array}{c}\text { Desire } \\
\text { Velocity } \\
\text { (rad/s) }\end{array}$} & Method & \multicolumn{2}{c}{$\begin{array}{c}\text { Reduction Percentage of } \\
\text { Link Velocity (\%) }\end{array}$} & \multicolumn{2}{c}{$\begin{array}{c}\text { Reduction Percentage of } \\
\text { Input Current (\%) }\end{array}$} \\
\cline { 3 - 6 } & & $\begin{array}{c}\text { Residual } \\
\text { Vibration }\end{array}$ & $\begin{array}{c}\text { Torque } \\
\text { Ripple }\end{array}$ & $\begin{array}{c}\text { Residual } \\
\text { Vibration }\end{array}$ & $\begin{array}{c}\text { Torque } \\
\text { Ripple }\end{array}$ \\
\hline \multirow{2}{*}{0.20} & ERBO & $26.7 \%$ & $5.2 \%$ & $32.1 \%$ & $6.3 \%$ \\
& SRC & $43.2 \%$ & $32.8 \%$ & $68.3 \%$ & $32.8 \%$ \\
& Proposed & $51.1 \%$ & $46.2 \%$ & $69.8 \%$ & $46.2 \%$ \\
\hline \multirow{2}{*}{0.33} & ERBO & $45.7 \%$ & $14.9 \%$ & $51.1 \%$ & $23.0 \%$ \\
& SRC & $62.9 \%$ & $70.2 \%$ & $86.1 \%$ & $91.2 \%$ \\
0.46 & Proposed & $70.2 \%$ & $76.3 \%$ & $93.2 \%$ & $91.2 \%$ \\
\hline \multirow{2}{*}{0.66} & ERBO & $52.1 \%$ & $47.6 \%$ & $52.6 \%$ & $48.3 \%$ \\
& SRC & $64.3 \%$ & $62.4 \%$ & $80.2 \%$ & $74.9 \%$ \\
& Proposed & $77.4 \%$ & $66.2 \%$ & $90.6 \%$ & $77.6 \%$ \\
\hline \multirow{2}{*}{0.85} & ERBO & $58.6 \%$ & $90.6 \%$ & $55.1 \%$ & $83.1 \%$ \\
& SRC & $68.5 \%$ & $81.3 \%$ & $78.9 \%$ & $73.8 \%$ \\
& Proposed & $81.5 \%$ & $81.3 \%$ & $85.0 \%$ & $73.8 \%$ \\
\hline & ERBO & $65.8 \%$ & no effect & $56.6 \%$ & no effect \\
& SRC & $74.0 \%$ & no effect & $70.6 \%$ & no effect \\
& Proposed & $86.3 \%$ & no effect & $80.1 \%$ & no effect \\
\hline
\end{tabular}

From experiment results, the torque ripple usually occurs during the low-speed uniform motion. When the twice-frequency of motion velocity overlaps the resonant frequency, the dominant vibration is caused by resonance modes. In this case, the torque ripple will submerge in noises. All in all, the proposed method has better suppression effects on these two kinds of vibrations than the ERBO method and SRC method.

\subsection{Robustness to Model Uncertainties}

In this section, the load inertia changes to act on the model uncertainties. When the load inertia fluctuates, the resonant frequency of the controlled system changes accordingly. To experimentally verify the robustness of model uncertainties, the load inertia can be changed by adding or subtracting the weights at the end of the connecting rod. When the load inertia increases $15 \%\left(J_{l}=2.60 \mathrm{~kg} \cdot \mathrm{m}^{2}\right)$ and decreases $15 \%\left(J_{l}=1.92 \mathrm{~kg} \cdot \mathrm{m}^{2}\right)$ from the initial value $\left(J_{l}=2.26 \mathrm{~kg} \cdot \mathrm{m}^{2}\right)$, the system resonant frequency decreases to $20.7 \mathrm{~Hz}$ and increases to $23.3 \mathrm{~Hz}$ accordingly. With the modular joint's dynamic parameters listed in Table 1 and the controller parameters listed in Table 2, repeated velocity tracking experiments from 0.33 to $0.85 \mathrm{rad} / \mathrm{s}$ under three different load inertias are shown in Figure 13.

After experimental verification, with the load inertia changing in the range of $J_{l} \pm 15 \%$, the proposed method still can suppress residual vibration and torque ripple. The effects of the proposed method contain in a band. Refer to the simulation analysis in Figure 10, the proposed method is robust to model uncertainties, which is simulated by the load inertia changing in experiments.

\subsection{Robustness to External Disturbances}

Moreover, to verify the robust performance to external disturbances, an impulse input current is triggered at $0.02 \mathrm{~s}$ with an amplitude of $6 \mathrm{~A}$ to act the external disturbances when the modular joint is held at the neutral position, as shown in Figure 14. 


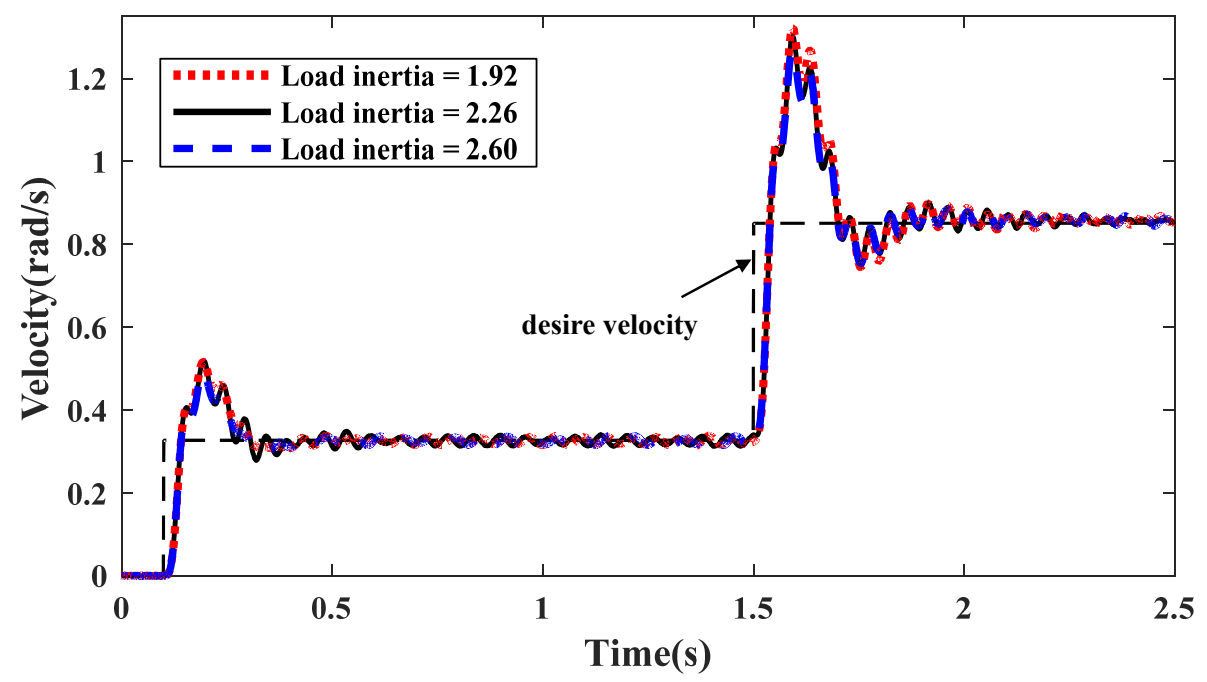

Figure 13. Robustness to model uncertainties experiments.

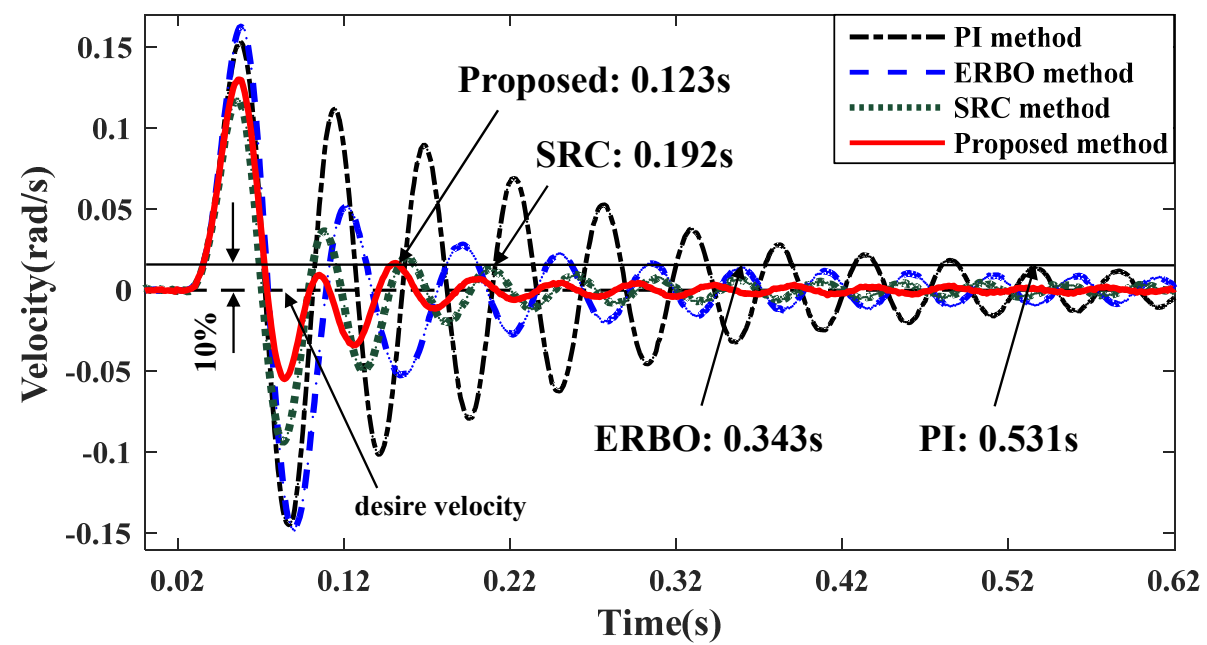

Figure 14. Robustness to external disturbances experiments.

When the fluctuation of link velocity is decaying within $10 \%$ after the impulse input current disturbing, the settling time of the PI method, ERBO method, SRC method, and the proposed method are $0.531 \mathrm{~s}, 0.343 \mathrm{~s}, 0.192 \mathrm{~s}$, and $0.123 \mathrm{~s}$, respectively. The percentage reduction in settling time with the ERBO method, SRC method, and proposed method with respect to the PI method are listed in Table 4.

Table 4. Reduction of settling time compared with the PI method.

\begin{tabular}{cccc}
\hline & $\begin{array}{c}\text { ERBO Method } \\
\mathbf{( \% )}\end{array}$ & $\begin{array}{c}\text { SRC Method } \\
\mathbf{( \% )}\end{array}$ & $\begin{array}{c}\text { Proposed Method } \\
\mathbf{( \% )}\end{array}$ \\
\hline $\begin{array}{c}\text { Percentage reduction } \\
\text { in settling time }\end{array}$ & $35.4 \%$ & $63.8 \%$ & $76.8 \%$ \\
\hline
\end{tabular}

Refer to the robust stability analysis in Figure 11, the proposed method is the most robust to external disturbances among these three methods, especially near the system resonant frequency. The experiments in Figure 14 show that the proposed method takes the least time to settle down, which confirms the theoretical analysis. 


\title{
6. Conclusions
}

A vibration controller is proposed to improve the modular joint's robustness to model uncertainties and external disturbances, confirmed by several relevant experiments. In this method, the equivalent rigid-body velocity is designed to be obtained by a high gain rigid-body state observer. The observer's high gain can significantly improve the robustness to model uncertainties and exotic disturbances, especially near the resonant frequency. Meanwhile, the high gain increases the bandwidth of the observer and the observation accuracy of the equivalent rigid-body velocity. In addition, an adjustable damper based on the high gain rigid-body state observer adds system damping, effectively reducing the residual vibration induced by the system resonant frequency during acceleration/deceleration and torque ripple caused by the harmonic drive during low-speed uniform motion simultaneously. Compared with a PI method and the other two rigid-body velocity methods-the ERBO method and the SRC method-the proposed method has better vibration suppression effects when the velocity changes abruptly. Moreover, it has superior velocity tracking accuracy during low-speed uniform motion. In the future, a fuzzy logic combined with the $H_{\infty}$ theory will be used to design the observer's gain and the adjustable damper's gain, allowing the controller to be adaptive to the load inertia changing in a wider range. Hence, the proposed method is expected to achieve vibration suppression in a manipulator whose posture changes, leading to the load inertia varying in real-time.

Author Contributions: Data curation, Q.X.; formal analysis, Q.X., C.W., and C.-Y.C.; funding acquisition, C.W., C.-Y.C., G.Y., and L.C.; investigation, Q.X., C.W., C.-Y.C., and G.Y.; methodology, Q.X.; project administration, G.Y. and L.C.; resources, Q.X., C.W., C.-Y.C., and G.Y.; software, Q.X. and C.W.; supervision, Q.X., C.-Y.C., G.Y., and L.C.; validation, Q.X.; visualization, Q.X.; writing_original draft, Q.X.; writing-review and editing, Q.X., C.-Y.C., and L.C. All authors have read and agreed to the published version of the manuscript.

Funding: This work was supported by the National Key R\&D Program of China (Grant No. 2019YFB1309900), the National Natural Science Foundation of China (Grant No. 51805523, U1813223, U1913214, U20A20282, 92048201), the International Partnership Program of the Chinese Academy of Sciences (Grant No. 174433KYSB20190036), the Key R\&D Program of Zhejiang Province (Grant No. 2021C01070, 2021C01067), the Ningbo Science and Technology Innovation 2025 Major Special Project (Grant No. 2018B10058).

Institutional Review Board Statement: Not applicable.

Informed Consent Statement: Not applicable.

Data Availability Statement: Not applicable.

Conflicts of Interest: The authors declare no conflict of interest.

\author{
Abbreviations \\ The following abbreviations are used in this manuscript: \\ HD Harmonic Drive \\ PI Proportional-Integral \\ ERBO Equivalent Rigid-Body Observer \\ SRC Self-Resonance Cancellation \\ DOB Disturbance Observer \\ ADRC Active Disturbance Rejection Control \\ ESO Extended State Observer \\ PMSM Permanent Magnet Synchronous Motor \\ ISE Integral of Squared Error criterion
}




\section{References}

1. Chan, M. Controller Synthesis and Vibration Suppression Techniques for Industrial Robotic Manipulators with Joint Flexibilities; UC Berkeley: Berkeley, CA, USA, 2013.

2. Xin, Q.; Chen, C.Y.; Wang, C.; Yang, G.; Zhang, C.; Fang, Z.; Chen, C.L.P. A Robust Method Based on Dual Encoders to Eliminate Velocity Ripple for Modular Drive Joints. Actuators 2020, 9, 135. [CrossRef]

3. Ozgoli, S.; Taghirad, H.D. A survey on the control of flexible joint robots. Asian J. Control 2006, 8, 332-344. [CrossRef]

4. Yuan, M.; Chen, Z.; Yao, B.; Hu, J. An Improved Online Trajectory Planner With Stability-Guaranteed Critical Test Curve Algorithm for Generalized Parametric Constraints. IEEE/ASME Trans. Mechatron. 2018, 23, 2459-2469. [CrossRef]

5. Béarée, R. New Damped-Jerk trajectory for vibration reduction. Control Eng. Pract. 2014, 28, 112-120. [CrossRef]

6. Cao, B.; Sun, K.; Li, T.; Gu, Y.; Jin, M.; Liu, H. Trajectory Modified in Joint Space for Vibration Suppression of Manipulator. IEEE Access 2018, 6, 57969-57980. [CrossRef]

7. Scoccia, C.; Palmieri, G.; Palpacelli, M.C.; Callegari, M. A Collision Avoidance Strategy for Redundant Manipulators in Dynamically Variable Environments: On-Line Perturbations of Off-Line Generated Trajectories. Machines 2021, 9, 30. [CrossRef]

8. Palmieri, G.; Scoccia, C. Motion Planning and Control of Redundant Manipulators for Dynamical Obstacle Avoidance. Machines 2021, 9, 121. [CrossRef]

9. Singhose, W. Command shaping for flexible systems: A review of the first 50 years. Int. J. Precis. Eng. Manuf. 2009, 10, 153-168. [CrossRef]

10. Singer, N.C.; Seering, W.P. Preshaping Command Inputs to Reduce System Vibration. J. Dyn. Syst. Meas. Control 1990, 112, 76-82. [CrossRef]

11. Park, J.; Chang, P.H.; Park, H.S.; Lee, E. Design of learning input shaping technique for residual vibration suppression in an industrial robot. IEEE/ASME Trans. Mechatron. 2006, 11, 55-65. [CrossRef]

12. Kim, J.; Croft, E.A. Preshaping input trajectories of industrial robots for vibration suppression. Robot. Comput. Integr. Manuf. 2018, 54, 35-44. [CrossRef]

13. Ahmad, M.A.; Suid, M.H.; Ramli, M.S.; Zawawi, M.A.; Ismail, R.R. PD Fuzzy Logic with non-collocated PID approach for vibration control of flexible joint manipulator. In Proceedings of the 2010 6th International Colloquium on Signal Processing and Its Applications, Malacca, Malaysia, 21-23 May 2010.

14. Ghazali, M.R.; Ibrahim, Z.; Suid, M.H.; Saealal, M.S.; Tumari, M. Single input fuzzy logic controller for flexible joint manipulator. Int. J. Innov. Comput. Inf. Control 2016, 12, 181-191.

15. Kelekci, E.; Kizir, S. Trajectory and vibration control of a flexible joint manipulator using interval type-2 fuzzy logic. ISA Trans. 2019, 94, 218-233. [CrossRef] [PubMed]

16. Cobanoglu, N.; Tatlicioglu, E.; Zergeroglu, E. Neural network based repetitive learning control of robot manipulators. In Proceedings of the 2017 American Control Conference, Seattle, WA, USA, 24-26 May 2017.

17. Chen, Z.; Wang, M.; Zou, Y. Dynamic learning from adaptive neural control for flexible joint robot with tracking error constraints using high-gain observer. Syst. Sci. Control Eng. Open Access J. 2018, 6, 177-190. [CrossRef]

18. He, W.; Yan, Z.; Sun, Y.; Ou, Y.; Sun, C. Neural-Learning-Based Control for a Constrained Robotic Manipulator With Flexible Joints. IEEE Trans. Neural Netw. Learn. Syst. 2018, 29, 5993-6003. [CrossRef]

19. Taghirad, H.D.; Rahimi, H. Composite QFT controller design for flexible joint robots. In Proceedings of the 2005 IEEE Conference on Control Applications, Toronto, ON, Canada, 28-31 August 2005.

20. Wang, Z.H.; Chen, Z.Q.; Sun, Q.L.; Yuan, Z.Z. Survey of the development for quantitative feedback theory. Control Theory Appl. 2006, 23, 403-410.

21. Aparna, V.; Banu, U.S.; Yasmin, S. PI controller design using quantitative feedback theory for a flow process. In Proceedings of the 2017 IEEE International Conference on Technological Innovations in Communication, Control and Automation, Chennai, India, 6 April 2017.

22. Radke, A.; Gao, Z. A survey of state and disturbance observers for practitioners. In Proceedings of the 2006 American Control Conference, Minneapolis, MN, USA, 14-16 June 2006.

23. Yun, J.N.; Su, J.; Kim, Y.I.; Kim, Y.C. Robust disturbance observer for two-inertia system. IEEE Trans. Ind. Electron. 2013, 60, 2700-2710. [CrossRef]

24. Chen, W.H.; Yang, J.; Guo, L.; Li, S. Disturbance-Observer-Based Control and Related Methods-An Overview. IEEE Trans. Ind. Electron. 2016, 63, 1083-1095. [CrossRef]

25. Yang, J.; Chen, W.H.; Li, S.; Guo, L.; Yan, Y. Disturbance/Uncertainty Estimation and Attenuation Techniques in PMSM Drives-A Survey. IEEE Trans. Ind. Electron. 2017, 64, 3273-3285. [CrossRef]

26. Huang, Y.; Xue, W. Active disturbance rejection control: Methodology and theoretical analysis. ISA Trans. 2014, 53, 963-976. [CrossRef] [PubMed]

27. Humaidi, A.J.; Badr, H.M.; Ajil, A.R. Design of Active Disturbance Rejection Control for Single-Link Flexible Joint Robot Manipulator. In Proceedings of the 2018 22nd International Conference on System Theory, Control and Computing (ICSTCC), Sinaia, Romania, 10-12 October 2018.

28. Hasegawa, A.; Fujimoto, H.; Takahashi, T. Robot joint angle control based on Self Resonance Cancellation using double encoders. In Proceedings of the 2017 IEEE International Conference on Advanced Intelligent Mechatronics (AIM), Munich, Germany, 3-7 July 2017. 
29. Yoshiura, Y.; Kawahara, S.; Horimai, D.; Asai, T.; Kaku, Y. Vibration Suppression Control Using an Equivalent Rigid-Body Observer. IEEJ J. Ind. Appl. 2018, 7, 396-402. [CrossRef]

30. Iwasaki, M.; Nakamura, H. Vibration Suppression for Angular Transmission Errors in Harmonic Drive Gearings and Application to Industrial Robots. Int. Fed. Autom. Control 2014, 47, 6831-6836. [CrossRef]

31. Xin, Q.; Wang, C.; Chen, C.Y.; Yang, G.; Fang, Z.; Chen, H. Vibration Suppression Method Based on the Equivalent Rigid Body State Observer of Modular Joint for a Collaborative Robot, presented at the Proceedings of the 17th International Conference on Manufacturing Research, Incorporating the 34th National Conference on Manufacturing Research; Queen's University Belfast: Belfast, UK, 2019.

32. Zhang, Q.; Liu, Z.; Xie, Z.; Yang, H.; Cai, H. Parameters Identification of Flexible Joints with Harmonic Driver. Jiqiren/Robot 2014, $36,164-170$

33. Dorf, R.; Bishop, R. Modern Control Systems, 12th ed.; Prentice Hall: Upper Saddle River, NJ, USA, 2011. 